\title{
Set existence principles and closure conditions: unravelling the standard view of reverse mathematics
}

\author{
Benedict Eastaugh*
}

February 16, 2018

\begin{abstract}
It is a striking fact from reverse mathematics that almost all theorems of countable and countably representable mathematics are equivalent to just five subsystems of second order arithmetic. The standard view is that the significance of these equivalences lies in the set existence principles that are necessary and sufficient to prove those theorems. In this article I analyse the role of set existence principles in reverse mathematics, and argue that they are best understood as closure conditions on the powerset of the natural numbers.
\end{abstract}

\section{Introduction}

What axioms are truly necessary for proving particular mathematical theorems? To answer this question, Harvey Friedman [1975, 1976] initiated a research program called reverse mathematics. By formalising ordinary mathematical concepts in the language of second order arithmetic, Friedman was able to show not only that many theorems of ordinary mathematics could be proved in relatively weak subsystems of second order arithmetic, but that such theorems often turned out to be equivalent to the axioms used to prove them, with this equivalence being provable in a weak base theory. Consider, for example, the least upper bound axiom for the real numbers. Not only are different formulations of the least upper bound axiom provably equivalent, but they are also provably equivalent to the axiom scheme of arithmetical comprehension. Reverse mathematics thus provides a unified framework within which one can precisely determine the strength of the axioms necessary to prove theorems of ordinary mathematics.

*Munich Center for Mathematical Philosophy, LMU Munich, Geschwister-Scholl-Platz 1, 80539 Munich, Germany. Email: benedict@eastaugh.net 
Reverse mathematics is carried out within subsystems of second order arithmetic, for which the canonical reference is Simpson [2009]. These subsystems are formulated in the language of second order arithmetic $\mathrm{L}_{2}$. This is a two-sorted first order language, the first sort of variables $x_{0}, x_{1}, \ldots$ being called number variables, and intended to range over natural numbers, and the second sort of variables $X_{0}, X_{1}, \ldots$ being called set variables and intended to range over sets of natural numbers. $L_{2}$ includes the non-logical symbols of first order Peano arithmetic (constant symbols 0 and 1, function symbols + and $\times$, and the relation symbol $<$ ), as well as a relation symbol $\in$ standing for set membership.

The system of full second order arithmetic or $\mathrm{Z}_{2}$ consists of: the basic axioms of Peano arithmetic minus the induction scheme, $\mathrm{PA}^{-}$; the $\Sigma_{1}^{0}$ induction scheme, consisting of the universal closures of all formulas of the form

$\left(\Sigma_{1}^{0}\right.$-IND)

$$
(\varphi(0) \wedge \forall n(\varphi(n) \rightarrow \varphi(n+1))) \rightarrow \forall n \varphi(n)
$$

where $\varphi(n)$ is a $\Sigma_{1}^{0}$ formula (which may contain free number and set variables); and the second order comprehension scheme, which consists of the universal closures of all formulas of the form

$\left(\Pi_{\infty}^{1}-\mathrm{CA}\right) \quad \exists X \forall n(n \in X \leftrightarrow \varphi(n))$

where $\varphi(n)$ is an $\mathrm{L}_{2}$-formula with $X$ not free (but possibly with other free number and set variables). $Z_{2}$ is, in the context of arithmetic, a very strong system, with consistency strength far beyond that of first order Peano arithmetic PA. The systems employed in reverse mathematics are all subsystems of $Z_{2}$, in the sense that all of their axioms are theorems of $Z_{2}{ }^{1}$

The most fundamental subsystem of second order arithmetic for reverse mathematics is $\mathrm{RCA}_{0}$. Its axioms are: the basic axioms; the $\Sigma_{1}^{0}$ induction scheme; and the recursive (or $\Delta_{1}^{0}$ ) comprehension scheme, which consists of the universal closures of all formulas of the form

$$
\forall n(\varphi(n) \leftrightarrow \psi(n)) \rightarrow \exists X \forall n(n \in X \leftrightarrow \varphi(n))
$$

where $\varphi$ is a $\Sigma_{1}^{0}$ formula, $\psi$ is a $\Pi_{1}^{0}$ formula, and $X$ is not free in $\varphi$ (although $\varphi$ may contain other free variables). It is the Recursive Comprehension Axiom that gives $\mathrm{RCA}_{0}$ its name. ${ }^{2} \mathrm{RCA}_{0}$ is the standard base theory for reverse mathematics: the system in which the equivalences between theorems and axioms are typically proved. In the rest of this article, unqualified expressions of the form " $\tau$ is equivalent to $S$ ", where $\tau$ is a mathematical theorem

\footnotetext{
${ }^{1}$ In order to achieve a streamlined and uniform presentation of $Z_{2}$ and its subsystems, this article deviates slightly from Simpson [2009], mainly in its treatment of induction axioms. This does not affect the results quoted in any material way.

${ }^{2}$ The ' 0 ' subscript indicates that this is a system with a restricted induction axiom, i.e. it does not prove the full second order induction scheme.
} 
and $S$ a subsystem of second order arithmetic, should be taken to mean that a faithful formalisation of $\tau$ can be proved in $\mathrm{RCA}_{0}$ to be implied by, and imply, the axioms of $S$. Similarly, " $\tau$ reverses to $S$ " means that a faithful formalisation of $\tau$ can be proved in $\mathrm{RCA}_{0}$ to imply the axioms of $S .{ }^{3}$ As $\mathrm{RCA}_{0}$ is the base theory for most reverse mathematics, the other systems considered in this article generally include the axioms of $\mathrm{RCA}_{0}$. The system $\mathrm{WKL}_{0}$, for example, consists of the axioms of $\mathrm{RCA}_{0}$ plus the axiom known as weak König's lemma, which states that every infinite subtree of the full binary tree $2^{<\mathbb{N}}$ has an infinite path through it. In such cases we say that the distinguishing axiom of $\mathrm{WKL}_{0}$ is weak König's lemma.

While there are many other subsystems of second order arithmetic, a particularly important group are known as the Big Five. As well as RCA and $\mathrm{WKL}_{0}$, this group consists of $A C \mathrm{~A}_{0}$, whose distinguishing axiom is the arithmetical comprehension scheme; $\mathrm{ATR}_{0}$, whose distinguishing axiom is the scheme of arithmetical transfinite recursion; and $\Pi_{1}^{1}-\mathrm{CA}_{0}$, whose distinguishing axiom is the $\Pi_{1}^{1}$-comprehension scheme. ${ }^{4}$ Each of these systems is stronger than the preceding systems, in the following sense: a system $S_{2}$ is proof-theoretically stronger than a system $S_{1}, S_{1}<S_{2}$, just in case $S_{2}$ proves all the theorems of $S_{1}$, but there is at least one theorem of $S_{2}$ that is not a theorem of $S_{1}$. For the Big Five we have that $\mathrm{RCA}_{0}<\mathrm{WKL}_{0}<\mathrm{ACA}_{0}<\mathrm{ATR}_{0}<\Pi_{1}^{1}-\mathrm{CA}_{0}$, although it should be noted that increases in proof-theoretic strength do not necessarily involve increases in consistency strength.

In most cases studied to date, ordinary mathematical theorems concerning countable and countably-representable objects have been found to be either provable in the base theory $\mathrm{RCA}_{0}$ or are equivalent over $\mathrm{RCA}_{0}$ to another of the Big Five. This is a remarkable phenomenon: Simpson [2010, p. 115] estimates that hundreds of theorems have been found that fall into these five equivalence classes. There is also is a substantial and growing body of statements that fall outside of this classification, known as the Reverse Mathematics Zoo [Dzhafarov, 2015]. These were originally drawn largely from infinitary combinatorics, by considering weakenings of Ramsey's theorem; an excellent starting point for the study of these statements is Hirschfeldt [2014]. However, the Zoo now contains examples from many other areas of mathematics including model theory, set theory, the theory of linear orderings, and descriptive set theory. Relatively few theorems from other core areas of mathematics such as analysis or algebra are known to lie outside the Big Five, although this situation is also changing: a recent example from analysis is Birkhoff's recurrence theorem [Day, 2016].

\footnotetext{
${ }^{3}$ The term "reversal" implies that the implication $S \Rightarrow \tau$ is already known, so I will not characterise implications $\tau \Rightarrow T$ where $T$ is proof-theoretically weaker than $\tau$ as "reversals"; instead I shall simply call them "implications".

${ }^{4}$ The historical development of these canonical five subsystems of second order arithmetic is traced in Dean and Walsh [2017].
} 
Explaining why the Big Five phenomenon occurs is an important task, and if an account of the significance of reversals could accomplish it, this would be a substantial accomplishment. However, it is also not one I take to be necessary for such accounts, and consequently I shall not address it directly in this article. One explanation offered by Montalbán [2011] is that the Big Five, and perhaps some other subsystems, are robust systems: they are invariant under certain perturbations of the axioms involved. Elaborations on this view can be found in Sanders [2013, 2015].

The standard view in the field of reverse mathematics is that the significance of reversals lies in their ability to demonstrate what set existence principles are necessary to prove theorems of ordinary mathematics. For example, they show that the arithmetical comprehension scheme is necessary in order to prove the least upper bound axiom for the real numbers, because the latter implies the former over the weak base theory RCA $A_{0}$ In contrast, arithmetical comprehension is not necessary in order to prove the Hahn-Banach theorem for separable Banach spaces, since this theorem can be proved in a weaker system than $\mathrm{ACA}_{0}$, namely $\mathrm{WKL}_{0}$. However, weak König's lemma is necessary to prove the separable Hahn-Banach theorem, and this necessity is demonstrated by the fact that $R C A_{0}$ proves that the latter implies the former. A view of this sort, in more or less the terms just used, is articulated by Simpson [2009, p. 2] as his "Main Question": "Which set existence axioms are needed to prove the theorems of ordinary, non-settheoretic mathematics?". Similar sentiments can be found elsewhere. ${ }^{5}$

It is not difficult to see why the standard view became and remains the orthodoxy, since in some sense the view can be simply read off from the mathematical results: each of the distinguishing axioms of the Big Five assert the existence of certain sets of natural numbers, and the hierarchy of proof-theoretic strength that we see in the Big Five can thus be understood as a hierarchy of set existence principles of increasing power. It also provides precise explanations of commonly expressed opinions such as "Gödel's completeness theorem is nonconstructive": the completeness theorem reverses to $\mathrm{WKL}_{0}$, which implies the existence of noncomputable sets, so any proof of the completeness theorem will necessarily employ a nonconstructive (in the sense of noncomputable) set existence principle. Finally, it allows us to comprehend within a common framework the various foundations for mathematics that can be faithfully formalised by subsystems of second order arithmetic as being differentiated (in terms of their mathematical consequences, rather than their philosophical justifications) by their commitment

\footnotetext{
${ }^{5}$ Such as in Friedman, Simpson, and Smith [1983, p. 141], Brown and Simpson [1986, p. 557], Jaeger [1987, p. 172], Brown, Giusto, and Simpson [2002, p. 191], Avigad and Simic [2006, p. 139] and Dorais, Dzhafarov, Hirst, Mileti, and Shafer [2016, p. 2]. There are many more examples to be found in the reverse mathematics literature, although it should be noted that many of the participants are students or coauthors of Simpson, and thus the similarity in language is not surprising.
} 
to set existence principles of differing strengths. Simpson [2010] proposes that each of the Big Five corresponds to a particular foundational program: $\mathrm{RCA}_{0}$ to computable mathematics; $\mathrm{WKL}_{0}$ to a partial realisation of Hilbert's program, as in Simpson [1988a]; ACA $_{0}$ to the predicative theory proposed by Weyl [1918]; ATR $\mathrm{A}_{0}$ to the predicative reductionist approach pioneered by Feferman [1964]; and $\Pi_{1}^{1}-\mathrm{CA}_{0}$ to the program of impredicative analysis found in Buchholz et al. [1981]. ${ }^{6}$

From the usual statement of the standard view, one might easily conclude that no ordinary mathematical theorems have been found that are not equivalent to particular set existence principles, but this is not the case. A formal version of Hilbert's basis theorem was shown by Simpson [1988b] to be equivalent to the wellordering of a recursive presentation of the ordinal $\omega^{\omega}$, while Rathjen and Weiermann [1993] showed that Kruskal's theorem is equivalent to the wellordering of a recursive presentation of $\vartheta \Omega^{\omega}$, the small Veblen ordinal. These wellordering statements are $\Pi_{1}^{1}$, and prima facie are not set existence principles. If anything, they are set nonexistence principles, stating that there exist no infinite sets witnessing the illfoundedness of the recursive linear orders representing the ordinals $\omega^{\omega}$ and $\vartheta \Omega^{\omega}$ respectively. Another class of examples is provided by the fragments of the first order induction scheme given by the $\Sigma_{n}^{0}$ induction and bounding schemes. Hirst [1987] showed that Ramsey's theorem for singletons, $\mathrm{RT}_{<\infty}^{1}$, is equivalent over $\mathrm{RCA}_{0}$ to the $\Sigma_{2}^{0}$ bounding scheme $\mathrm{B} \Sigma_{2}^{0}$. Equivalences to stronger and weaker fragments of first order induction are also proved in Simpson and Smith [1986] and Friedman, Simpson, and Yu [1993]. These cases demonstrate that the standard view should not be interpreted as the claim that all equivalences proved in reverse mathematics concern set existence principles. Rather, in cases where a reversal from theorem $\tau$ to a subsystem $S$ of second order arithmetic show that a set existence principle is required to prove $\tau$, the reversal demonstrates which set existence principle is required.

Since the base theory $\mathrm{RCA}_{0}$ is both expressively impoverished and prooftheoretically weak, the coding involved in representing ordinary mathematical objects (such as real numbers, complete separable metric spaces, Borel and analytic sets, and so on) in that theory is substantial. For reversals to have the significance ascribed to them by the standard view requires that the process of formalisation is faithful, in the sense that the formal statements in the language of second order arithmetic formally capture the mathematical content of statements of ordinary mathematics. In what follows, we shall take this faithfulness for granted, at least for the specific mathematical theorems discussed. Nevertheless, there are cases where we might doubt this faithfulness, or at least worry about the commitments that attend certain types of coding, as in the investigations of Kohlenbach [2002] and Hunter

\footnotetext{
${ }^{6}$ Further references for these connections can be found in Simpson [2010], while a more thoroughgoing analysis of them appears in Dean and Walsh [2017].
} 
[2008].

Despite its appeal, the standard view as it has been advanced in the literature to date has a major weakness, namely that its central concept of a set existence principle is left unanalysed, and thus the precise content of the view is unclear. The goal of this article is therefore to provide such an analysis. Our starting point is to note that accounts of the significance of reversals may offer at least two kinds of explanation. The first is the significance of reversals as a general matter: what do the equivalences proved in reverse mathematics between theorems of ordinary mathematics and canonical subsystems of second order arithmetic tell us? Let us call such explanations "global". The second is the significance of reversals to a particular system, such as the significance of reversals to $\mathrm{WKL}_{0}$ discussed above. Let us call explanations of this sort "local". Depending on the account one offers, local explanations may follow from global explanations, in part or in whole, or they may not. The standard view is, prima facie, committed to a global explanation of the significance of reversals, namely that they allow us to calibrate the strength of set existence principles necessary to prove theorems of ordinary mathematics. However, without a clear understanding of what set existence principles are, it is unclear what the relationship between local and global explanations is under the standard view. For example, it is commonly held that reversals to $\mathrm{WKL}_{0}$ demonstrate that proofs of the given theorem must involve some form of compactness argument. In itself this is a local explanation, but without a fuller account of what set existence principles are, it is left open whether it follows from the global explanation of the significance of reversals in terms of set existence principles, or whether it is genuinely local in character. As we shall see, the answer to this question is highly sensitive to the particular account of set existence principles one adopts.

\section{Set existence as comprehension}

One initially attractive way of cashing out the notion of a set existence principle is taking it to be identical to the concept of a comprehension scheme. I call this view "set existence as comprehension" or SEC. A comprehension scheme is a schematic principle, each instance of which asserts the existence of the (uniquely determined, by extensionality) set of all and only those objects that satisfy a given predicate. In the case of second order arithmetic, the objects are natural numbers, and the predicates concerned are formulas of the language $\mathrm{L}_{2}$ of second order arithmetic. Typically, a comprehension scheme collectively asserts the existence of the sets defined by a particular formula class, as follows. Let $\Gamma$ be a set of formulas in the language $\mathrm{L}_{2}$ of second order arithmetic. The $\Gamma$-comprehension scheme consists of the 
universal closures of all formulas of the form

$$
\exists X \forall n(n \in X \leftrightarrow \varphi(n))
$$

where $\varphi$ belongs to $\Gamma$ and $X$ is not free in $\varphi$. If $\Gamma$ consists of all $\mathrm{L}_{2}$-formulas, we obtain the full comprehension scheme $\Pi_{\infty}^{1}$-CA of second order arithmetic, the distinguishing axiom of full second order arithmetic $Z_{2}$. By restricting $\Gamma$ to different formula classes, we obtain different comprehension schemes. For example, if $\Gamma$ consists of formulas with only number quantifiers, we obtain the arithmetical comprehension scheme, the distinguishing axiom of the subsystem $\mathrm{ACA}_{0}$. By restricting to formulas of the form $\exists k \theta(k)$ where $\theta$ is quantifier-free ( $\Sigma_{1}^{0}$ formulas), we obtain the $\Sigma_{1}^{0}$-comprehension scheme; this is in fact equivalent to the arithmetical comprehension scheme. By restricting to formulas of the form $\forall X \theta(X)$ where $\theta$ is arithmetical, we obtain the $\Pi_{1}^{1}$-comprehension scheme, the distinguishing axiom of the subsystem $\Pi_{1}^{1}-C_{0}$. Some comprehension schemes do not follow this template to the letter, most notably the recursive comprehension scheme $\left(\Delta_{1}^{0}-\mathrm{CA}\right)$. This is a subscheme of $\Sigma_{1}^{0}$-comprehension incorporating the further restriction that each admissible formula be not merely $\Sigma_{1}^{0}$, but provably equivalent to a $\Pi_{1}^{0}$ formula (i.e. of the form $\forall k \theta(k)$ where $\theta$ is quantifier-free).

The idea that any given formal property (i.e. one defined by a formula of a formal language properly applied to some domain) has an extension is a highly credible basic principle, so long as appropriate precautions are taken to avoid pathological instances. Second order arithmetic is a fragment of simple type theory, which guards against Russell-style paradoxes, as formulas like $X \notin X$ are not well-formed and thus cannot appear in instances of comprehension. Moreover, comprehension schemes fall into straightforward hierarchies, with increasingly strong comprehension schemes permitting the use of syntactically broader classes of definitions. This harmonises with the fact that some theorems are true even of the computable sets, while others entail the existence of witnesses that are computability-theoretically highly complex, and strong axioms are therefore needed in order to prove them. Such gradations can also be seen as hierarchies of acceptability: if one denies that noncomputable sets exist then recursive comprehension forms a natural stopping point; if one repudiates impredicativity then arithmetical comprehension could be a good principle to adopt; and so on.

Of the Big Five subsystems of second order arithmetic which are of primary importance to reverse mathematics, only three are characterised by comprehension schemes: $R C A_{0}, A C A_{0}$ and $\Pi_{1}^{1}-C A_{0}$. The intermediate systems $\mathrm{WKL}_{0}$ and $A T R_{0}$ are obtained by adding further axioms (weak König's lemma in the former case, arithmetical transfinite recursion in the latter) to a comprehension scheme. Nevertheless, one might think that while weak König's lemma and arithmetical transfinite recursion are not formulated as comprehension schemes, they are nonetheless equivalent to comprehension 
schemes. The following result of Dean and Walsh shows that this is not the case for weak König's lemma. ${ }^{7}$

Theorem 2.1 (Dean and Walsh, private communication). No subset of the arithmetical comprehension scheme is equivalent over $\mathrm{RCA}_{0}$ to weak König's lemma.

Proof. Assume for a contradiction that there is a set of arithmetical formulas $\Psi$ such that $\mathrm{RCA}_{0}$ proves that $\Psi$-CA is equivalent to weak König's lemma. Without loss of generality, we may assume that there is a single arithmetical formula $\varphi(n, X)$ with only the displayed free variables such that

$$
\mathrm{RCA}_{0} \vdash \mathrm{WKL} \leftrightarrow \forall X C_{\varphi}(X),
$$

where $C_{\varphi}(X)$ is the instance of arithmetical comprehension asserting that the set $\{n \in \mathbb{N} \mid \varphi(n, X)\}$ exists.

Let $M$ be a countable $\omega$-model of $\mathrm{WKL}_{0}$, and thus of $\forall X C_{\varphi}(X)$. WKL is computably false, so there exist $X, Y \in M$ with $Y=\{n \in \omega \mid \varphi(n, X)\}$ and $X \lessgtr_{\mathbf{T}} Y$. By cone avoidance for $\Pi_{1}^{0}$ classes, there exists a countable $\omega$-model $M^{\prime}$ of $\mathrm{WKL}_{0}$ such that $X \in M^{\prime}$ but $Y \notin M^{\prime}$. Since $M^{\prime}$ must also be a model of $C_{\varphi}(X)$, there exists $Z \in M^{\prime}$ such that $n \in Z \leftrightarrow \varphi(n, X)$ for all $n \in \omega$. But then by extensionality $Z=Y$, contradicting the fact that $Y \notin M^{\prime}$.

Dean and Walsh [2017, pp. 29-30] argue that weak König's lemma is therefore a counterexample to SEC. Crucially, $\mathrm{WKL}_{0}$ is not merely a subsystem of second order arithmetic that is not equivalent to a comprehension scheme: it is a mathematically natural one, since weak König's lemma is equivalent over $\mathrm{RCA}_{0}$ to the Heine-Borel covering lemma, Brouwer's fixed point theorem, the separable Hahn-Banach theorem, and many other theorems of analysis and algebra.

Simpson [2010, p. 119] defines a subsystem of second order arithmetic as being mathematically natural just in case it is equivalent over a weak base theory to one or more "core" mathematical theorems. $\mathrm{WKL}_{0}, \mathrm{ACA}_{0}$, $\mathrm{ATR}_{0}$ and $\Pi_{1}^{1}-\mathrm{CA}_{0}$ are all mathematically natural systems, since each one is equivalent over $\mathrm{RCA}_{0}$ to many theorems from different areas of ordinary mathematics. This is a notion that seems to admit of degrees: some systems may, in virtue of being equivalent to many core mathematical theorems, be more mathematically natural than those which are only equivalent to a few

\footnotetext{
${ }^{7}$ Dean and Walsh's proof of this theorem appeals to Simpson, Tanaka, and Yamazaki [2002]'s result that $\mathrm{WKL}_{0}$ is conservative over $\mathrm{RCA}_{0}$ for sentences of the form $\forall X \exists ! Y \varphi(X, Y)$, where $\varphi$ is arithmetical. The theorem also follows from the non-existence of minimal $\omega$-models of $\mathrm{WKL}_{0}$ and, as in the proof I give here, from basis theorems for $\Pi_{1}^{0}$ classes (Diamondstone, Dzhafarov, and Soare [2010] is a good reference for such basis theorems). My thanks to the two anonymous referees for their suggestions towards a more optimal proof.
} 
such theorems. When a claim of the form " $S$ is a mathematically natural system" is used in an unqualified way in the rest of this article, it should be taken to mean that $S$ meets the minimum requirement of being equivalent to at least one core mathematical theorem. The notion of mathematical naturalness appears to give us a partial answer to the question of the significance of reversals: by proving an equivalence between a theorem of ordinary mathematics $\tau$ and a subsystem of second order arithmetic $S$, we thereby demonstrate that $S$ is a mathematically natural system. However, this still leaves us in the dark about the significance of the reversal for the theorem $\tau$ : what important property of this theorem of ordinary mathematics do we come to know when we prove its equivalence over a weak base theory to $S$, that we did not know before? It is this question that the standard view, and thus the SEC account as an explication of the standard view's central theoretical notion, attempts to answer.

Dean and Walsh's argument that SEC fails runs as follows: since weak König's lemma is neither a comprehension scheme, nor equivalent to one, it cannot be a set existence principle (as by SEC, set existence principles are just comprehension schemes). So the significance of a reversal from a theorem $\tau$ to weak König's lemma cannot lie in the comprehension scheme that is both necessary and sufficient to prove it, since there is no such scheme. Either the significance of reverse mathematics does not lie in the set existence principles which theorems reverse to, or the set existence as comprehension view is false. Proponents of the standard view are thus on shaky ground. They must adopt a more sophisticated way of spelling out their core contention, or abandon the idea that the significance of reversals lies in set existence principles. We can also understand Dean and Walsh's point in terms of the distinction between local and global explanations: SEC fails to offer a local explanation of the significance of reversals to weak König's lemma, since it is not a comprehension scheme, despite the fact that reversals to $\mathrm{WKL}_{0}$ are clearly significant. The global explanation of the significance of reversals offered by the SEC account is therefore inadequate.

One response to Dean and Walsh's argument is to endorse a more expansive conception of set existence principles, based on the fact that Weak König's lemma and arithmetical transfinite recursion are equivalent to schematic principles of a different sort, namely separation schemes. The separation scheme for a class of formulas $\Gamma$ holds that if two formulas $\varphi, \psi \in \Gamma$ are provably disjoint, then there exists a set whose members include every $n \in \mathbb{N}$ such that $\varphi(n)$, and exclude every $n \in \mathbb{N}$ such that $\psi(n)$. Weak König's lemma is equivalent over $\mathrm{RCA}_{0}$ to $\Sigma_{1}^{0}$-separation, while arithmetical transfinite recursion is equivalent over $\mathrm{RCA} \mathrm{A}_{0}$ to $\Sigma_{1}^{1}$-separation.

Definition 2.2 (separation scheme). Let $\Gamma$ be a class of formulas, possibly containing free variables. The $\Gamma$-separation scheme, $\Gamma$-SEP, consists of the 
universal closures of all formulas of the form

$(\Gamma$-SEP $) \forall n(\neg(\varphi(n) \wedge \psi(n))) \rightarrow \exists X \forall n((\varphi(n) \rightarrow n \in X) \wedge(\psi(n) \rightarrow n \notin X))$,

where $\varphi, \psi \in \Gamma$ with $X$ not free.

One response to Dean and Walsh's argument is to endorse the following more expansive conception of set existence principles: both comprehension schemes and separation schemes are set existence principles.

In line with our existing terminology, we call the proposal that both comprehension schemes and separation schemes are set existence principles SECS. This solves the immediate problem with the SEC view, since each of the Big Five are equivalent over $\mathrm{RCA}_{0}$ to either a comprehension scheme or a separation scheme. But although SECS accommodates both weak König's lemma and arithmetical transfinite recursion, and thus evades the counterexamples that sink SEC, it does so at the price of a seemingly ad hoc modification to the view.

\section{Conceptual constraints}

The arguments levelled against the SEC account and its variants tacitly appeal to different constraints which the concept of a set existence principle should satisfy, if it is to play a role in explaining the significance of reversals. I shall now attempt to make these constraints explicit, by presenting three conditions which any satisfactory account of the concept of a set existence principle should meet, together with some reasons to believe that these conditions are plausible. I shall then show how the SECS account meets two of the stated conditions, but fails to satisfy a third. In the following we take $\mathcal{A}$ to be some account of the concept of a set existence principle (e.g. SEC, SECS) with an associated extension $\mathcal{S}(\mathcal{A})$, consisting of the set of subsystems of second order arithmetic which, according to the account $\mathcal{A}$, express set existence principles.

(1) Nontriviality. Not all subsystems of second order arithmetic are classified as set existence principles by $\mathcal{A}$, i.e. at least one subsystem is not a member of $\mathcal{S}(\mathcal{A})$.

(2) Comprenensiveness. If $S$ is a mathematically natural subsystem of second order arithmetic, and $S$ actually expresses a set existence principle, then $\mathcal{A}$ classifies $S$ as expressing a set existence principle: $S \in \mathcal{S}(\mathcal{A})$.

(3) UnITy. The subsystems of second order arithmetic which $\mathcal{A}$ classifies as expressing set existence principles, i.e. the members of $\mathcal{S}(\mathcal{A})$, are conceptually unified. 
Consider some account of set existence principles $\mathcal{A}$. Such an account should lend substance to the claim that the significance of reversals lies in the set existence principles necessary to prove theorems of ordinary mathematics, by providing some degree of analysis of the concept of a set existence principle, and a way to determine whether a subsystem of second order arithmetic expresses a set existence principle (for example, the SEC account does this by stating that set existence principles are just comprehension schemes). If $\mathcal{A}$ does not satisfy the nontriviality condition (3) then it cannot do this. There are many statements of second order arithmetic, such as purely arithmetical ones containing no set quantifiers, that prima facie are not set existence principles. Violating the nontriviality condition therefore entails failing to provide a theory that is truly an account of set existence principles at all.

If $\mathcal{A}$ does not meet the comprehensiveness condition (3), then it not only fails to include prima facie set existence principles, but ones that are equivalent to core mathematical theorems. $\mathcal{A}$ would therefore fail to provide a satisfactory reconstruction of the concept of a set existence principle in reverse mathematics, and the reconstruction offered would be unable to play its intended role in the standard view. The antecedent of the condition is relatively strong, since it requires that a system both express a set existence principle and be mathematically natural. As noted in $\S 1$, some core mathematical theorems reverse to principles that are prima facie not set existence principles, such as Hilbert's basis theorem which is equivalent over $\mathrm{RCA}_{0}$ to $\mathrm{WO}\left(\omega^{\omega}\right)$. It is thus not to $\mathcal{A}$ 's detriment - and, indeed, may be to its credit - if it does not classify such systems as set existence principles. On the other hand, there are also systems that prima facie express set existence principles, but that are not mathematically natural, or at least are not known to be. It is reasonable, but not required, for $\mathcal{A}$ to include such systems. The $\Delta_{1}^{1}$-comprehension scheme is a set existence principle according to the SEC account, but it has not been proved equivalent to any theorem of ordinary mathematics. Various forms of the axiom of choice, such as the systems $\Sigma_{1}^{1}$-AC and $\Sigma_{1}^{1}$-DC, also appear to be set existence principles that are not mathematically natural, but in these cases the SEC account does not classify them as set existence principles. ${ }^{8}$ The comprehensiveness condition (3) permits such cases because in order for an account of the concept of a set existence principle to elucidate the standard view of reverse mathematics, it does not need to say anything about the status of systems which are not equivalent to any theorem of ordinary mathematics. With these points in mind, we can see clearly why the fact that SEC does not include weak König's lemma is so problematic. Resisting its mathematical naturalness is difficult, since it is equivalent to dozens of core mathematical theorems in analysis, algebra, logic, and combinatorics. Moreover, it is prima facie a set

\footnotetext{
${ }^{8}$ See definition VII.6.1 of Simpson [2009, p. 294] for details of these systems.
} 
existence principle, albeit in a somewhat different sense to comprehension schemes. The standard view that reversals demonstrate the set existence axioms necessary to prove theorems of ordinary mathematics would not be the standard view in the field if it did not include $\mathrm{WKL}_{0}$.

Any failure of $\mathcal{A}$ to meet the unity condition (3) has a somewhat different character. The standard view is an attempt to provide a general account of the significance of reversals, one that does not make overt reference to particular systems. Such generality requires that the systems which $\mathcal{A}$ countenances as set existence principles have some features in common. For example, while recursive comprehension, arithmetical comprehension and $\Pi_{1}^{1}$-comprehension are all different, the SEC account still satisfies the unity condition precisely because they are all comprehension schemes, and it can offer a theory under which all comprehension schemes can legitimately be considered to be set existence principles. If $\mathcal{A}$ does not satisfy the unity condition then it cannot be considered as offering a satisfactory theory of set existence principles, even if it is extensionally adequate in the sense of satisfying conditions (3) and (3). If no account meeting this condition can be found then we are reduced to merely offering local explanations of the significance of reversals to particular systems, rather than a global explanation of the significance of reverse mathematical results. Moreover, an inability to provide a unified account of the concept of a set existence principle would cast doubt on whether there is indeed a unified concept that can play the explanatory role the standard view requires of it.

Unity, however, comes in degrees, and so accounts of the concept of a set existence principle can satisfy it in a stronger or weaker way. When there are strong connections between the different systems considered to be set existence principles, then the account under which those systems are considered to be set existence principles can be said to strongly satisfy the unity condition. In such cases it seems reasonable to expect that the significance of reversals to a particular system $S$ will, in large part, be given in terms of the account of set existence principles, rather than in terms of specific properties of $S$ that are at substantial variance to other set existence principles. The SEC account exhibits this property: different comprehension schemes are learly the same type of principle, and can be obtained by simple syntactic restrictions on a stronger principle, namely the full comprehension scheme. evertheless, requiring that any theory of set existence principles satisfies the unity condition as strongly as the SEC account is an onerous requirement that may well be impossible to meet in a theory that also satisfies the comprehensiveness condition. Allowing for theories to satisfy the unity requirement only weakly, and have different set existence principles bear a mere family resemblance to one another, rather than be strictly of the same type of axiom in some strong syntactic sense, seems a reasonable relaxation of the ondition.

Since the comprehensiveness condition (3) is a conditional and not a bi- 
conditional, it does not require that every subsystem that expresses a set existence principle according to $\mathcal{A}$ also expresses set existence principle by the lights of our naïve, informal, or intuitive understanding of the concept. This reflects the kind of counterexample encountered in $\S 2$ and $\S 4$, namely WKL and WWKL. These are sentences that prima facie express set existence principles, but are not classified as such, by the SEC and SECS accounts respectively. Contrastingly, we have few examples of sentences that are classified as set existence principles by the accounts currently on the table, and yet prima facie do not express set existence principles. ${ }^{9}$ Moreover, such cases do not seem to undermine the coherence or usefulness of the concept of a set existence principle in the way that counterexamples like WKL and WWKL do, because they do not give us the same sense that our theory of set existence principles is somehow inadequate to the data. The nontriviality condition (3) and the unity condition (3) are therefore formulated so as to constrain how extensive the class of subsystems classified as set existence principles by $\mathcal{A}$ is, but the conditions as a whole do not require that $\mathcal{A}$ provide a list of subsystems that is coextensive with our informal understanding of the concept of a set existence principle.

\section{Set existence as comprehension and separation}

Admitting separation schemes as set existence principles is, prima facie, an ad hoc modification of the SEC view that weakens one of the main strengths of the SEC view, namely its strong satisfaction of the unity condition (3). The primary point of difference between separation and comprehension schemes is that as straightforward definability axioms, comprehension schemes tell us which particular sets exist. Separation schemes, on the other hand, do not always do so: an axiom asserting the mere existence of a separating set may not pin down a particular set as the witness for this assertion. A striking theorem in this vein is that the only sets which every $\omega$-model of $\Sigma_{1}^{0}$-separation (i.e. $\mathrm{WKL}_{0}$ ) has in common are the computable ones. This illustrates Friedman [1975, p. 235]'s point that "Much more is needed to define explicitly a hard-to-define set of integers than merely to prove their existence."

To rebut the charge that SECS is ad hoc, and show that it does after all satisfy the unity requirement, we must show that there is some degree of conceptual commonality between comprehension schemes and separation schemes. Let us first note that one important comprehension scheme is a separation scheme: $\Delta_{1}^{0}$-comprehension is equivalent, over a weak base theory, to the $\Pi_{1}^{0}$-separation scheme. Moreover, following Lee [2014], we can treat the entire Big Five in a unified way by understanding them as interpolation schemes.

\footnotetext{
${ }^{9}$ Induction axioms are one possible exception; see footnote 13 for details.
} 
Definition 4.1 (interpolation scheme). Let $\Gamma$ and $\Delta$ be sets of $\mathrm{L}_{2}$-formulas, possibly with free variables. The $\Gamma-\Delta$ interpolation scheme, $\Gamma-\Delta$-INT, consists of the universal closures of all formulas of the form

$$
\begin{aligned}
& \forall m(\varphi(m) \rightarrow \psi(m)) \\
\rightarrow & \exists X \forall m((\varphi(m) \rightarrow m \in X) \wedge(m \in X \rightarrow \psi(m)))
\end{aligned}
$$

where $\varphi \in \Gamma$ and $\psi \in \Delta$, and $X$ is not free in either formula.

All of the Big Five are equivalent to interpolation schemes: $\mathrm{RCA}_{0}$ is equivalent to $\Pi_{1}^{0}-\Sigma_{1}^{0}$-INT; $\mathrm{WKL}_{0}$ to $\Sigma_{1}^{0}$ - $_{1}^{0}$-INT; $\mathrm{ACA} \mathrm{A}_{0}$ to $\Sigma_{1}^{0}$ - $\Sigma_{1}^{0}$-INT; $\mathrm{ATR} \mathrm{R}_{0}$ to $\Sigma_{1}^{1}-\Pi_{1}^{1}$-INT; and $\Pi_{1}^{1}-\mathrm{CA}_{0}$ to $\Sigma_{1}^{1}-\Sigma_{1}^{1}$-INT. This should go at least some way towards ameliorating our worry that SECS fails to satisfy the unity condition (3), since we can now see that both comprehension schemes and separation schemes are actually interpolation schemes.

Mere syntactic unity should not by itself convince us of the conceptual unity of comprehension schemes and separation schemes; after all, a sufficiently broad syntactically specified class of sentences will eventually contain all statements. The notion of an interpolation scheme is, however, relatively narrow and it is not hard to see that it is a straightforward generalisation of the concepts of separation and comprehension. The comprehension scheme for some class of formulas $\Phi$ can be derived from the $\Phi-\Phi$ interpolation scheme, since for any instance of comprehension we can use the given formula in both places in the interpolation scheme and thus derive the comprehension instance. Separation schemes, on the other hand, arise when given some formula class $\Delta$, the formula class $\Gamma$ consists of the negations of the formulas in $\Delta$, such as when $\Delta=\Sigma_{1}^{0}$ and $\Gamma=\Pi_{1}^{0} \cdot{ }^{10}$

However, even if we grant that SECS satisfies the unity condition (3), it still fails to offer a satisfactory theory of set existence principles, since there is a mathematically natural counterexample which shows that it does not satisfy the comprehensiveness condition (3), namely the axiom known as weak weak König's lemma. This principle was introduced in Yu [1987], and as the name suggests, it is a further weakening of weak König's lemma, obtained by restricting weak König's lemma to trees with positive measure.

Definition 4.2 (weak weak König's lemma). Weak weak König's lemma (WWKL) is the statement that if $T$ is a subtree of $2^{<\mathbb{N}}$ with no infinite path, then

$$
\lim _{n \rightarrow \infty} \frac{|\{\sigma \in T \mid \operatorname{lh}(\sigma)=n\}|}{2^{n}}=0 .
$$

\footnotetext{
${ }^{10}$ As noted in $\S 2, \mathrm{RCA}_{0}$ deviates somewhat from the standard template for comprehension schemes, and for this reason is not equivalent to an interpolation scheme of the type $\Phi-\Phi$-INT where both sets of formulas are the same. By analogy with the case of RCA $\mathrm{A}_{0}$, one might expect the $\Delta_{1}^{1}$-comprehension scheme to be equivalent to $\Pi_{1}^{1}-\Sigma_{1}^{1}$-INT, i.e. the $\Pi_{1}^{1}$-separation scheme. However, this is not the case, as Montalbán [2008] proved. Since $\Delta_{1}^{1}-C A_{0}$ is not known to be a mathematically natural subsystem of $Z_{2}$, this fact does not by itself seem to pose a particular problem for the SECS view.
} 
The system $W W K L_{0}$ is given by adjoining WWKL to the axioms of $R C A_{0}$.

$\mathrm{WWKL}_{0}$ is strictly intermediate between $\mathrm{RCA}_{0}$ and $\mathrm{WKL}_{0}[\mathrm{Yu}$ and Simpson, 1990], and is equivalent over $\mathrm{RCA}_{0}$ to a number of theorems from measure theory, such as a formal version of the Vitali Covering Theorem [Brown, Giusto, and Simpson, 2002]; the countable additivity of the Lebesgue measure [Yu and Simpson, 1990]; and the monotone convergence theorem for the Lebesgue measure on the closed unit interval. A survey of results in this area is given in Simpson [2009, §X.1]. These equivalences show that WWKL is mathematically natural in Simpson's sense. By the comprehensiveness condition (3) we should therefore expect a good account of set existence principles to include it.

$W W K L_{0}$ is not equivalent over $\mathrm{RCA}_{0}$ to any subset of the arithmetical comprehension scheme. ${ }^{11} \mathrm{Yu}$ and Simpson [1990, $\S 2$, pp. 172-3] proved that not every model of $W W K L_{0}$ is a model of $W K L_{0}$. Their proof involves the construction of what is known as a random real model, and the properties of this model show that $W_{W K L_{0}}$ is not equivalent over $R C A_{0}$ to any subscheme of $\Sigma_{1}^{0}$-separation. Since $W W K L_{0}$ is not a separation scheme, it is a mathematically natural subsystem of $Z_{2}$ that SECS cannot accommodate. SECS therefore fails to satisfy the comprehensiveness condition (3).

Theorem 4.3 (Yu and Simpson 1990). Weak weak König's lemma is not equivalent over $\mathrm{RCA}_{0}$ to any subscheme of the $\Sigma_{1}^{0}$-separation scheme.

A virtue that it would be reasonable to expect of any account of set existence principles is the ability to incorporate the discovery of new subsystems of second order arithmetic which turn out to be equivalent to theorems of ordinary mathematics. Banking on SEC or its extensions amounts to a bet that all such new systems will be comprehension schemes or separation schemes. The discovery of WWKL and the role it plays in the reverse mathematics of measure theory shows that such optimism is unfounded even for the mathematically natural systems which are already known. In the next section I will advance an account of set existence principles which does not suffer from this weakness.

\section{Closure conditions}

In a sense the term "set existence principles" is an unfortunate one, since it implies that the relevant principles simply assert the existence of some sets, independently of the other axioms of the theory. A better term, which more accurately captures the nature of these axioms, is "closure conditions". These are not bare or unconditional statements of set existence, but conditional principles. In general they hold that given the existence of a set

\footnotetext{
${ }^{11}$ This follows, for example, from the conservativity theorem of Simpson, Tanaka, and Yamazaki [2002] mentioned in footnote 7.
} 
$X \in \mathcal{P}(\mathbb{N})$ with certain properties, there exists another set $Y \in \mathcal{P}(\mathbb{N})$ standing in some relation to $X$. Weak König's lemma, for example, asserts that $\mathcal{P}(\mathbb{N})$ is closed under the existence of paths through infinite subtrees of $2^{<\mathbb{N}}$. This is a conditional set existence principle since it requires that there be such trees in the first place. In the absence of other suitable existence axioms, weak König's lemma alone would not allow us to prove the existence of any sets at all, and is thus a set existence principle only relative to other axioms such as recursive comprehension.

On the other hand, comprehension schemes appear at first blush to be set existence principles tout court. Nevertheless, they too are better understood as closure conditions, because the comprehension schemes used in reverse mathematics all admit parameters. Comparing the standard formulation of recursive comprehension (in which parameters are allowed) with the parameter-free version makes this clear. The parameter-free recursive comprehension scheme asserts the existence of those sets definable in a $\Delta_{1}^{0}$ way, without reference to any other sets. But recursive comprehension with parameters instead asserts that $\mathcal{P}(\mathbb{N})$ is closed under relative recursiveness: if $X \subseteq \mathbb{N}$ exists, so does every $Y \leq_{\mathbf{T}} X$. While comprehension schemes do have a different flavour to other closure conditions, they can often be characterised in equivalent ways which more closely hew to the model described above for weak König's lemma. Arithmetical comprehension, for example, is equivalent over $\mathrm{RCA}_{0}$ to König's lemma, the statement that every finitely branching infinite subtree of $\mathbb{N}<\mathbb{N}$ has an infinite path through it. $\Pi_{1}^{1}-C_{0}$ is equivalent over $\mathrm{RCA}_{0}$ to the statement that for every tree $T \subseteq \mathbb{N}<\mathbb{N}$, if $T$ has a path then it has a leftmost path.

With these points in mind, I propose that the best way to understand the concept of a set existence principle in reverse mathematics is by means of the concept of a closure condition on the powerset of the natural numbers. Reformulating the standard view of reverse mathematics in these terms, we come to the view that the significance of a provable equivalence between a theorem of ordinary mathematics $\tau$ and a subsystem $S$ of second order arithmetic lies in telling us what closure conditions $\mathcal{P}(\mathbb{N})$ must satisfy in order for $\tau$ to be true. This is a bit of a mouthful, so we shall adopt the following slogan as an abbreviation for the view: reversals track closure conditions.

Views of this sort have been stated elsewhere, most notably by Feferman [1992, p. 451] who identifies set existence principles with closure conditions in his discussion of the mathematical existence principles justified by empirical science. Indeed, already in Feferman [1964, p. 8] we find that work in the spirit of Weyl's predicative analysis "isolates various closure conditions on a collection of real numbers which are necessary to obtain [results such as the Bolzano-Weierstraß and Heine-Borel theorems], in this case closure under arithmetical definability." Similar positions have also been taken in the reverse mathematics literature, for example by Dorais, Dzhafarov, Hirst, 
Mileti, and Shafer [2016, p. 2], who write that each subsystem studied in reverse mathematics "corresponds to a natural closure point under logical, and more specifically, computability-theoretic, operations", and by Chong, Slaman, and Yang [2014, p. 864], who write that "Ultimately, we are attempting to understand the relationships between closure properties of $2^{\mathbb{N}}$ ". Shore [2010] also states that the Big Five correspond to recursion-theoretic closure conditions: this is clearly something in the air. However, none of these authors make precise what they mean by a closure condition, nor draw out the consequences of this view, although Chong, Slaman, and Yang [2014] consider it to have consequences for the practice of reverse mathematics, for instance in showing that $\omega$-models have a particular importance.

In order to clarify the content of the view that reversals track closure conditions, let us distinguish two things: a closure condition in itself, and the different axiomatizations of that closure condition. Closure conditions are extensional: they are conditions on the powerset which assert that it is closed in some way, for example under arithmetical definability. Axiomatisations of closure conditions are intensional: one and the same closure condition will admit of infinitely many different axiomatizations. For example, the Turing jump operator gives rise to a closure condition, of which some of the better-known axiomatizations are (modulo the base theory $\mathrm{RCA}_{0}$ ): the arithmetical comprehension scheme; König's lemma; and the BolzanoWeierstraß theorem.

The upshot of this distinction is that by proving reversals we show that different theorems of ordinary mathematics correspond to the same closure conditions. The significance of reversals thus lies, at least to a substantial extent, in placing these theorems in a hierarchy of well-understood closure conditions of known strength. Note also that there is a duality here: an equivalence between a theorem $\tau$ and a system $S$ tells us something about $\tau$, namely its truth conditions in terms of what closure condition must hold for it to be true, but it also tells us something about the closure condition itself, namely how much of ordinary mathematics is true in $\mathcal{P}(\mathbb{N})$ when that closure condition holds.

This account incorporates a substantial relativism to the base theory, in at least two distinct ways. The first is that, as noted above, some closure conditions such as weak König's lemma are set existence principles only in a conditional sense, and using them to prove the existence of sets relies on them being used in conjunction with other axioms. The second is that the equivalences which I take to show that different statements express the same closure condition must be proved in some base theory. Typically, this will be the usual base theory for reverse mathematics, $\mathrm{RCA}_{0}$, but in some cases a stronger base theory is required in order to prove that two statements are equivalent and thus express the same closure conditions.

The view that reversals track closure conditions has some marked advantages over the SEC account and its variants such as SECS. Most notably, it 
can accommodate all of the counterexamples discussed so far. Weak König's lemma is a closure condition, and thus we improve on the SEC account; but so is weak weak König's lemma, and thus the new account also succeeds where the SECS account fails. Other principles which have been studied in reverse mathematics - arithmetical transfinite recursion, choice schemes, and many others - can all be understood as expressing closure conditions on $\mathcal{P}(\mathbb{N})$. Moreover, this account will also accommodate any similar principle discovered to be equivalent to a theorem of ordinary mathematics. In order to determine whether or not the view goes beyond this apparent improvement over the SEC and SECS accounts, and provides a satisfactory account of the significance of reversals in general, we return to the three conditions that I argued in $\S 3$ any account of set existence principles should satisfy: nontriviality (3), comprehensiveness (3), and unity (3).

By analysing the notion of a set existence principle in terms of closure conditions on the powerset of the natural numbers, the account clearly offers a unified picture of what set existence principles are. However, the notion of a closure condition is a very general one. At first glance, the view seems committed to the idea that every $\Pi_{2}^{1}$ sentence expresses a closure condition. By way of contrast, the specificity of the concept of a comprehension scheme means that the SEC account strongly satisfies the unity condition. But this very feature undermines its suitability as an analysis of the concept of a set existence principle, since it fails to be sufficiently comprehensive, as the existence of striking counterexamples such as weak König's lemma illustrates. We therefore must conclude that although the view that reversals track closure conditions satisfies the unity condition, it only satisfies it in a relatively weak sense. As such, it is reasonable to wonder to what degree the view can offer a compelling explanation of the significance of reversals, since if it is easy for an $\mathrm{L}_{2}$-sentence to be considered as expressing a closure condition, then it is unclear what light this can shed on the importance of particular reversals. There is, however, a clear sense in which all closure conditions are the same kind of thing: if weak König's lemma and Ramsey's theorem for pairs are not in exactly the same class of principles, they certainly bear a family resemblance to one another. With this in mind I contend that weakly satisfying the unity condition is sufficient to make the view that reversals track closure conditions a viable account, and moreover that weakly satisfying this condition is all one can expect of an account of set existence principles that accommodates not only WKL and WWKL, but also the constellation of combinatorial and model-theoretic statements in the Reverse Mathematics Zoo.

The generality of the view also undermines, to some extent, its ability to satisfy the nontriviality condition (3). Arithmetical statements cannot be considered as expressing closure conditions, and thus according to the account they do not express set existence principles. Neither do $\Pi_{1}^{1}$ statements, such as those expressing that a given recursive ordinal $\alpha$ is wellordered. 
Nevertheless, it is hard to escape from the conclusion that at least every $\Pi_{2}^{1}$ statement should be considered a closure condition. After all, it is the very form of these statements - which assert that for every set $X \subseteq \mathbb{N}$ of a certain sort, there exists a set $Y \subseteq \mathbb{N}$ of a different sort - that brought us to consider the view that reversals track closure conditions in the first place.

This point holds more generally: thus far we have only considered closure conditions with $\Pi_{2}^{1}$ formulations, but not even all of the Big Five have $\Pi_{2}^{1}$ definitions. In particular, $\Pi_{1}^{1}-C_{0}$ is not equivalent over $A T R_{0}$ to any $\Pi_{2}^{1}$ statement, although it is straightforwardly expressed as a $\Pi_{3}^{1}$ sentence. There are even theorems that exceed the strength of $\Pi_{1}^{1}$-comprehension, such as "Every countably based MF space which is regular is homeomorphic to a complete separable metric space", which is equivalent to $\Pi_{2}^{1}$-comprehension [Mummert and Simpson, 2005]. Such theorems are not expressible as $\Pi_{3}^{1}$ statements, so we must consider yet more complex sentences as also expressing closure conditions if we are to bring them into the account.

When we restrict our attention to the closure conditions expressed by $\Pi_{2}^{1}$ sentences, the conditions involved are arithmetical, and thus indicate that $\mathcal{P}(\mathbb{N})$ is closed under the existence of sets which are definable without quantification over the totality of sets of natural numbers. In other words, $\Pi_{2}^{1}$ closure conditions are predicative. On an intuitive level, we can understand these predicative closure conditions as local conditions, in the following sense. Suppose we have a class of sets $\mathcal{C} \subseteq \mathcal{P}(\mathbb{N})$ and a closure condition which states that for every $X$ such that $\psi(X)$, there exists a $Y$ such that $\varphi(X, Y)$, where $\psi$ and $\varphi$ are arithmetical. If there is such an $X \in \mathcal{C}$ such that there is no corresponding $Y \in \mathcal{C}$, then $\mathcal{C}$ does not satisfy the instance of the closure condition for $X$, and must be expanded to a class $\mathcal{C}^{\prime} \supseteq \mathcal{C}$ such that $Y \in \mathcal{C}^{\prime}$ for some $Y$ such that $\varphi(X, Y)$. Satisfying the instance of the closure condition for $X$ thus depends only on the existence of $Y$, and the arithmetical properties of $X$ and $Y$. For impredicative set existence principles such as $\Pi_{1}^{1}$ or $\Pi_{2}^{1}$ comprehension, the situation is more complex, since if a class of sets $\mathcal{C}$ fails to satisfy such a principle, then the new sets that must be added to $\mathcal{C}$ in order to satisfy it depend not just on the single set $X$, but also on the entirety of elements of the expanded class $\mathcal{C}^{\prime} \supseteq \mathcal{C}$. Impredicative closure conditions are thus global conditions rather than local ones. The more general, impredicative notion of closure at work here is thus distinct from, and stronger than, that which applies in the case where the conditions are arithmetical. Nevertheless, it is still recognisably a notion of closure: an impredicative closure condition requires that for every set $X$ there exists a $Y$ bearing some relation to $X$, although that relation may be given in terms of not just arithmetical properties of $X$ and $Y$, but properties of all sets of natural numbers.

Taking these considerations to their natural conclusion, it seems that we should grant that all $\Pi_{n}^{1}$ sentences, for $n \geq 2$, express closure conditions (for brevity's sake we shall sometimes refer to such sentences as $\Pi_{n \geq 2}^{1}$ sentences 
in the remainder of this article). Sentences of lower complexity (for example, arithmetical sentences) can be transformed into $\Pi_{2}^{1}$ sentences by the addition of dummy quantifiers. To work around this difficulty, we impose the following constraint on the $\Pi_{n>2}^{1}$ sentences which we take to express closure conditions. For all $n \geq 1$, we say that a sentence $\varphi$ is essentially $\Pi_{n}^{1}$ (respectively, essentially $\Sigma_{n}^{1}$ ) if, over an appropriate base theory $B$, it is equivalent to a sentence that is $\Pi_{n}^{1}$ (respectively, $\Sigma_{n}^{1}$ ), and not equivalent to any $\Sigma_{n}^{1}$ sentence (respectively, $\Pi_{n}^{1}$ ), or to any sentence of lower complexity. The base theory $B$ should not prove $\varphi$, but otherwise it should be as strong as possible. ${ }^{12}$ These requirements are intended to ensure that if a sentence $\varphi$ does not express a closure condition, then a sufficiently strong base theory is available to prove this fact. For example, consider the following sentence $\psi$ : "WKL $\wedge 0^{\prime}$ exists". $\psi$ is $\Pi_{2}^{1}$, but not essentially $\Pi_{2}^{1}$. While $\mathrm{RCA}_{0}$ does not prove $\psi$, neither does $\mathrm{WKL}_{0}$, since weak König's lemma does not imply the existence of the Turing jump. However, since $W_{K L}$ proves the first conjunct, it proves that $\psi$ is equivalent to the $\Sigma_{1}^{1}$ sentence " $0^{\prime}$ exists". $\psi$ is thus not essentially $\Pi_{2}^{1}$, and does not express a closure condition. This seems like the correct outcome, since $\psi$ only asserts that $0^{\prime}$ exists, rather than making the more general (and essentially $\Pi_{2}^{1}$ ) claim that for all $X, X^{\prime}$ exists. With the notion of essentially $\Pi_{n}^{1}$ and $\Sigma_{n}^{1}$ sentences in hand, we can give a more precise account of which sentences express closure conditions, namely all and only the essentially $\Pi_{n \geq 2}^{1}$ sentences.

Dean and Walsh [2017, pp. 29-30] express the worry that there might be no broader notion of set existence principle that includes both comprehension schemes and principles like weak König's lemma and arithmetical transfinite recursion, beyond that of accepting all $\Pi_{2}^{1}$ sentences as expressing set existence principles. As argued in $\S 4$, there is a broader notion (the SECS view) that includes weak König's lemma and arithmetical transfinite recursion, but this broader notion remains vulnerable to mathematically natural counterexamples like WWKL. In endorsing the view that all and only the essentially $\Pi_{n \geq 2}^{1}$ sentences express closure conditions, I am biting this bullet and broadly concurring with Dean and Walsh that there is no viable broader notion of set existence principle beyond that encompassing all essentially $\Pi_{2}^{1}$ sentences (and worse, all essentially $\Pi_{n}^{1}$ sentences as well, for $n \geq 3$ ). Since the essentialness restriction rules out all sentences in the class $\Sigma_{1}^{1} \cup \Pi_{1}^{1}$, as well as many others, the situation is not disastrous: measuring set existence is not "just the same as sorting out the very fine-grained equivalence classes of mutual derivability" [Dean and Walsh, 2017, p. 30], although it is not so far away from this either.

The restriction to essentially $\Pi_{n \geq 2}^{1}$ sentences also rules out wellordering

\footnotetext{
${ }^{12}$ The term "essentially $\Pi_{n}^{1}\left(\Sigma_{n}^{1}\right)$ formula" is used in the literature on subsystems of second order arithmetic to denote a different, formally specified formula class (e.g. Simpson [2009, definition VIII.6.1, p. 348]). In this paper, however, the term "essentially $\Pi_{n}^{1}\left(\Sigma_{n}^{1}\right)$ sentence" will only be used in the sense of the definition just given.
} 
statements such as $\mathrm{WO}\left(\omega^{\omega}\right)$, which seems like the correct outcome, but other cases are less clear-cut. According to Simpson [2009, remark II.3.11, p. 71-2], $\Sigma_{n}^{0}$-induction is a set existence principle, because it is equivalent to bounded $\Sigma_{n}^{0}$-comprehension (i.e. comprehension for finite $\Sigma_{n}^{0}$-definable sets). However, since $\Sigma_{n}^{0}$-induction can (for any $n \in \omega$ ) be axiomatized by a $\Pi_{1}^{1}$ sentence, it does not express a closure condition, and hence according to the present account it is not a set existence principle. Moreover, we can also run Simpson's argument in reverse: since bounded $\Sigma_{n}^{0}$-comprehension is equivalent to $\Sigma_{n}^{0}$-induction, it does not (contrary to appearances) express a set existence principle after all. As induction axioms have a very different character to the closure conditions we have been considering in the rest of this paper, it seems reasonable to consider them as distinct kinds of axiom. Simpson appears to classify induction axioms as set existence principles primarily to further bolster his claim that reverse mathematics shows us what set existence principles are necessary to proving theorems of ordinary mathematics. In light of the necessity of axioms such as $\mathrm{WO}\left(\omega^{\omega}\right)$ to proving results like Hilbert's basis theorem, it does not seem overly problematic to take the line that reverse mathematics shows that not only set existence principles, but also other kinds of axiom, are required in order to prove theorems of ordinary mathematics. Amongst these we can include both wellordering statements and induction axioms. ${ }^{13}$

A different kind of difficulty is posed by parameter-free comprehension schemes. These assert that a certain class of definable sets exist; for example, the parameter-free $\Sigma_{1}^{0}$-comprehension scheme asserts that all sets exist that are definable by a $\Sigma_{1}^{0}$ formula without free set variables. Because of the existence of universal $\Sigma_{1}^{0}$ sets, the parameter-free $\Sigma_{1}^{0}$-comprehension scheme can (in the presence of recursive comprehension, with parameters) be axiomatized by a single $\Sigma_{1}^{1}$ formula. More generally, parameter-free comprehension schemes are axiomatized by essentially $\Sigma_{n \geq 1}^{1}$ sentences. They thus appear to be set existence principles which are not closure conditions, since they do not assert that (for example) $\mathcal{P}(\mathbb{N})$ is closed under the existence of $\Sigma_{1}^{0}$ definable sets, but merely that all sets definable by $\Sigma_{1}^{0}$ formulas without set parameters exist.

This presents a challenge to the view defended in this section: if not all set existence principles are closure conditions, then the claim that I have provided an analysis of the notion of a set existence principle in terms of closure conditions is in jeopardy. The obvious response to this worry is that parameter-free comprehension schemes are not mathematically natural:

\footnotetext{
${ }^{13}$ One lacuna is induction schemes where the formula class for which induction is permitted is not arithmetical, such as $\Sigma_{1}^{1}$-induction. These appear to be equivalent to essentially $\Pi_{n \geq 2}^{1}$ sentences, and thus express closure conditions. This is an unfortunate asymmetry with the arithmetical induction scheme and its subschemes such as $\Sigma_{2}^{0}$-induction. This asymmetry can be overcome by requiring that all base theories include full induction, or by only considering $\omega$-models as Shore [2010] does, but these approaches incur other costs.
} 
they are not equivalent to ordinary mathematical theorems. This is clearly a defeasible claim - a core mathematical theorem equivalent to parameterfree $\Sigma_{1}^{0}$-comprehension could be found tomorrow, after all - and moreover, although parameter-free comprehension schemes are not equivalent to ordinary mathematical theorems as studied in reverse mathematics, they are often equivalent to particular instances of those theorems. For instance, the parameter-free $\Sigma_{1}^{0}$-comprehension scheme is equivalent to the restriction of the Bolzano-Weierstraß theorem to a particular computable, bounded sequence of real numbers.

This line of argument should be resisted. While in a narrow, technical sense any derivable sentence is a theorem, in ordinary mathematical practice we do not grant this appellation so lightly. We have strong antecedent reasons to hold that there is a difference in degree, if not in kind, between theorems on the one hand, and mere facts on the other. The BolzanoWeierstraß theorem is more informative, more general, more deep, and more useful than its instances, considered individually or even collectively: unlike them, it is deservedly called a theorem. I do not intend to precisely articulate the distinction between theorems and mere mathematical facts-I simply note that it is a distinction in mathematical practice that, absent compelling evidence to the contrary, we should treat as a substantial conceptual distinction with attendant explanatory power. On the basis of this distinction, $\Sigma_{1}^{0}$-comprehension with parameters is mathematically natural, while $\Sigma_{1}^{0}$-comprehension without parameters is not. The restriction in the comprehensiveness constraint (3) to mathematically natural principles reflects the aim of the present paper, namely to determine what kinds of theories can stand as explications of the concept of a set existence principle as it appears in reverse mathematics, rather than in mathematics or logic in general. With this in mind, the fact that parameter-free comprehension schemes are not equivalent to core mathematical theorems means that they should not be considered to be counterexamples to the analysis of set existence principles as closure conditions on $\mathcal{P}(\mathbb{N})$.

By striking a balance closer to triviality than noncomprehensiveness, the view that reversals track closure conditions accommodates the most central part of reverse mathematics, namely the study of mathematically natural $\Pi_{2}^{1}$ theorems. Although such a general account does not, by itself, offer substantial local explanations of the significance of particular reversals, it does at least offer a framework within which more fine-grained theorising can be done. The explanatory power offered by SEC can be partially recovered by acknowledging that some closure conditions are comprehension schemes, and that comprehension schemes are a class of principles with distinctive qualities, such that their necessary use in the proof of an ordinary mathematical theorem will allow distinctive kinds of explanation. Other classes of principles, such as separation schemes, may also allow for explanations of the significance of reversals to any system in that class. 
On this version of the standard view, all that can be said in general about the significance of the equivalences to set existence principles proved in reverse mathematics is that they show that crucial theorems in diverse areas of ordinary mathematics require that $\mathcal{P}(\mathbb{N})$ satisfies particular closure conditions. These closure conditions can be captured by natural axioms with an arguably logical character. An individual reversal demonstrates the closure condition required to support a given part of ordinary mathematics, and in some sense picks out an intrinsic feature of a theorem, namely the resources required to prove it, whether that be compactness or transfinite recursion. This feature is a proof-invariant property: every proof of the theorem in question must at some point make use of this property, although it may appear in different guises.

\section{Funding}

This work was supported by the Arts and Humanities Research Council [doctoral studentship, 2011-14]; and the Leverhulme Trust [International Network grant "Set Theoretic Pluralism"].

\section{Acknowledgements}

This article is drawn from my doctoral research at the University of Bristol, and builds builds on work by Walter Dean and Sean Walsh which was unpublished during the writing of this article, but which has now appeared in part as [Dean and Walsh, 2017]. I would like to thank them for their generosity in sharing their research, and their helpful comments on my own. I would also like to thank Marianna Antonutti Marfori, Michael Detlefsen, Leon Horsten, Øystein Linnebo, Toby Meadows, Richard Pettigrew, and Sam Sanders, for valuable discussions of the topics addressed herein; the audiences of talks at Helsinki, Leuven, London, and Munich, where I presented earlier versions of this material; and the editor and two anonymous referees, whose comments helped sharpen this paper considerably.

\section{References}

J. Avigad and K. Simic. Fundamental Notions of Analysis in Subsystems of Second-Order Arithmetic. Annals of Pure and Applied Logic, 139(1-3), 2006. doi:10.1016/j.apal.2005.03.004.

D. K. Brown and S. G. Simpson. Which set existence axioms are necessary to prove the separable Hahn-Banach theorem? Annals of Pure and Applied Logic, 31:123-144, 1986. doi:10.1016/0168-0072(86)90066-7. 
D. K. Brown, M. Giusto, and S. G. Simpson. Vitali's Theorem and WWKL. Archive for Mathematical Logic, 41(2):191-206, 2002. doi:10.1007/s001530100100.

W. Buchholz, S. Feferman, W. Pohlers, and W. Sieg, editors. Iterated Inductive Definitions and Subsystems of Analysis: Recent Proof-Theoretical Studies, volume 897 of Lecture Notes in Mathematics. Springer Berlin Heidelberg, 1981. doi:10.1007/BFb0091894.

C. T. Chong, T. A. Slaman, and Y. Yang. The metamathematics of Stable Ramsey's Theorem for Pairs. Journal of the American Mathematical Society, 27(3):863-892, 2014. doi:10.1090/S0894-0347-2014-00789-X.

A. R. Day. On the strength of two recurrence theorems. The Journal of Symbolic Logic, 81(4):1357-1374, 2016. doi:10.1017/jsl.2016.9.

W. Dean and S. Walsh. The prehistory of the subsystems of secondorder arithmetic. The Review of Symbolic Logic, 10(2):357-396, 2017. doi:10.1017/S1755020316000411.

D. E. Diamondstone, D. D. Dzhafarov, and R. I. Soare. $\Pi_{1}^{0}$ Classes, Peano Arithmetic, Randomness, and Computable Domination. Notre Dame Journal of Formal Logic, 51(1):127-159, 2010. doi:10.1215/002945272010-009.

F. G. Dorais, D. D. Dzhafarov, J. L. Hirst, J. R. Mileti, and P. Shafer. On uniform relationships between combinatorial problems. Transactions of the American Mathematical Society, 368:1321-1359, 2016. doi:10.1090/tran/6465.

D. D. Dzhafarov. The Reverse Mathematics Zoo. http://rmzoo.math. uconn. edu, 2015. Accessed February 6, 2018.

S. Feferman. Systems of predicative analysis. The Journal of Symbolic Logic, 29(1):1-30, 1964. doi:10.2307/2269764.

S. Feferman. Why a Little Bit Goes a Long Way: Logical Foundations of Scientifically Applicable Mathematics. In D. Hull, M. Forbes, and K. Okruhlik, editors, PSA: Proceedings of the Biennial Meeting of the Philosophy of Science Association, volume 1992, Volume Two: Symposia and Invited Papers, pages 442-455. The University of Chicago Press on behalf of the Philosophy of Science Association, 1992. doi:10.1086/psaprocbienmeetp.1992.2.192856.

H. Friedman. Some Systems of Second Order Arithmetic and Their Use. In Proceedings of the 17th International Congress of Mathematicians, Vancouver 1974, volume 1, pages 235-242, 1975. 
H. Friedman. Systems of second order arithmetic with restricted induction. I, II. The Journal of Symbolic Logic, 41(2):557-559, 1976. doi:10.1017/S0022481200051665. (Abstracts).

H. Friedman, S. G. Simpson, and X. Yu. Periodic points and subsystems of second-order arithmetic. Annals of Pure and Applied Logic, 62:51-64, 1993. doi:10.1016/0168-0072(93)90187-I.

H. M. Friedman, S. G. Simpson, and R. L. Smith. Countable algebra and set existence axioms. Annals of Pure and Applied Logic, 25(2):141-181, 1983. doi:10.1016/0168-0072(83)90012-X.

D. R. Hirschfeldt. Slicing the Truth: On the Computability Theoretic and Reverse Mathematical Analysis of Combinatorial Principles. Number 28 in Lecture Notes Series, Institute for Mathematical Sciences, National University of Singapore. World Scientific Publishing Company, 2014.

J. L. Hirst. Combinatorics in Subsystems of Second Order Arithmetic. PhD thesis, Pennsylvania State University, August 1987.

J. L. Hunter. Higher-Order Reverse Topology. PhD thesis, University of Wisconsin-Madison, 2008. URL http://www.math.wisc.edu/ lempp/ theses/hunter.pdf. Accessed February 15, 2018. iv + 97 pages.

G. Jaeger. Some Proof-Theoretic Contributions to Theories of Sets. In T. P. L. Group, editor, Logic Colloquium '85, volume 122 of Studies in Logic and the Foundations of Mathematics, pages 171-191. North-Holland, Amsterdam, 1987. doi:10.1016/S0049-237X(09)70553-1.

U. Kohlenbach. Foundational and mathematical uses of higher types. In W. Sieg, R. Sommer, and C. Talcott, editors, Reflections on the Foundations of Mathematics: Essays in Honor of Solomon Feferman, volume 15 of Lecture Notes in Logic, pages 92-116. A. K. Peters, 2002. doi:10.1017/9781316755983.005.

G. Lee. The big five systems of reverse mathematics and their computational interpretation. Presented at CORCON 2014, Genoa, 2014. Work in progress with Hugo Herbelin and Keiko Nakata.

A. Montalbán. On the $\Pi_{1}^{1}$-separation principle. Mathematical Logic Quarterly, 54:563-578, 2008. doi:10.1002/malq.200710049.

A. Montalbán. Open questions in reverse mathematics. The Bulletin of Symbolic Logic, 17(3):431-454, 2011. doi:10.2178/bsl/1309952320.

C. Mummert and S. G. Simpson. Reverse mathematics and $\Pi_{2}^{1}$ comprehension. The Bulletin of Symbolic Logic, 11(4):526-533, 2005. doi:10.2178/bsl/1130335208. 
M. Rathjen and A. Weiermann. Proof-theoretic investigations on Kruskal's theorem. Annals of Pure and Applied Logic, 60(1):49-88, 1993. doi:10.1016/0168-0072(93)90192-G.

S. Sanders. Reverse-engineering Reverse Mathematics. Annals of Pure and Applied Logic, 164(5):528-541, May 2013. doi:10.1016/j.apal.2012.11.006.

S. Sanders. The taming of the Reverse Mathematics zoo. arXiv:1412.2022v6, 2015 .

R. A. Shore. Reverse Mathematics: The Playground of Logic. The Bulletin of Symbolic Logic, Volume 16, Number 3:378-402, 2010. doi:10.2178/bsl/1286284559.

S. G. Simpson. Partial realizations of Hilbert's program. The Journal of Symbolic Logic, 53:349-363, 1988a. doi:10.1017/S0022481200028309.

S. G. Simpson. Ordinal numbers and the Hilbert basis theorem. Journal of Symbolic Logic, 53(3):961-974, 1988b. doi:10.2307/2274585.

S. G. Simpson. Subsystems of Second Order Arithmetic. Cambridge University Press, Cambridge, 2nd edition, 2009. doi:10.1017/cbo9780511581007.

S. G. Simpson. The Gödel hierarchy and reverse mathematics. In S. Feferman, C. Parsons, and S. G. Simpson, editors, Kurt Gödel, Essays for His Centennial, volume 33 of Lecture Notes in Logic, pages 109-127. Cambridge University Press, 2010. doi:10.1017/CBO9780511750762.008.

S. G. Simpson and R. L. Smith. Factorization of polynomials and $\Sigma_{1}^{0}$ induction. Annals of Pure and Applied Logic, 31:289-306, 1986. doi:10.1016/0168-0072(86)90074-6.

S. G. Simpson, K. Tanaka, and T. Yamazaki. Some conservation results on weak König's lemma. Annals of Pure and Applied Logic, 118(1-2):87-114, 2002. doi:10.1016/S0168-0072(01)00121-X.

H. Weyl. Das Kontinuum: Kritische Untersuchungen über die Grundlagen der Analysis. Veit, 1918. Reprinted in: H. Weyl, E. Landau, and B. Riemann, Das Kontinuum und andere Monographien, Chelsea, 1960, 1973.

X. Yu. Measure Theory in Weak Subsystems of Second Order Arithmetic. $\mathrm{PhD}$ thesis, Pennsylvania State University, 1987. vii +73 pages.

X. Yu and S. G. Simpson. Measure theory and weak König's lemma. Archive for Mathematical Logic, 30:171-180, 1990. doi:10.1007/BF01621469. 